\title{
Development of SCAR Markers for DNA Fingerprinting and Germplasm Analysis of American Cranberry
}

\author{
James J. Polashock ${ }^{1}$ and Nicholi Vorsa ${ }^{2}$ \\ Philip E.Marucci Center for Blueberry and Cranberry Research, Rutgers The State University of New Jersey, \\ 125A Lake Oswego Road, Chatsworth, NJ 08019
}

\begin{abstract}
AdDiTIONAL INDEX woRDs. Vaccinium macrocarpon, multiplex PCR, genetic relatedness, Cy5, genotyping, fluorescent, RAPD markers

AbSTRACT. DNA fingerprinting has been useful for genotypic classification of American cranberry (Vaccinium macrocarpon Ait.). Polymerase chain reaction (PCR) based methodologies including randomly amplified polymorphic DNA (RAPD) markers are relatively easy to use, and inexpensive as compared to other methods. However, RAPD markers have some limitations including seamless interlaboratory transferability and susceptibility to certain types of error. An alternative method, sequence characterized amplified regions (SCARs), was developed for cranberry germplasm analysis. Nine primer sets were designed from RAPD-identified polymorphic markers for use in two multiplex PCR reactions. These primer sets generated 38 markers across a cranberry germplasm collection. Estimates of genetic relatedness deduced from employment of the RAPD and SCAR methods were compared among 27 randomly chosen cranberry germplasm accessions. Although both methods produced comparable results above 0.90 coefficient of similarity, branches below this level exhibited variation in clustering. SCAR and RAPD markers can be employed for identifying closely related genotypes. However, the inferences of more distant genetic relationships are less certain. SCAR marker reactions provided more polymorphic markers on a per reaction basis than RAPD marker reactions and as such more readily separated closely related progeny. When SCAR primers were fluorescent dye-labeled for computerized detection and data collection, reduced marker intensity relative to unlabeled reactions was one problem encountered.
\end{abstract}

Cultivated beds of American cranberry (Vacciniummacrocarpon) are generally started using pressed-in vines or stem cuttings harvested from established beds. Newly planted beds typically take 2 to 3 years to reach full production potential and can remain productive for many years. Most cultivars are clonal, but some older cultivars appear to have been mixtures of genotypes when originally selected from the wild (Polashock and Vorsa, unpublished). Evidence suggests that over time, encroachment by wild vines or seedlings and unproductive volunteer seedlings from self- or crosspollinations become established in cultivated beds, lowering yield potential (Novy et al., 1996). Since cranberry lacks qualitative morphological characters to allow for visual classification, an alternative method of genotype identification is necessary. Since beds of most cultivars are supposed to be pure clones but are often contaminated as noted above, a genotyping method would be useful in determining if a problem such as poor yield has a genetic (i.e., due to the establishment of a contaminating genotype) or environmental basis. For breeding purposes, confirmation of genetic identity and estimating genetic similarity would be valuable.

Since large sample numbers must be processed for any of the purposes listed above, a rapid and reliable method is required. The method of choice should provide for good genome coverage, be relatively simple to use, and be cost effective. Random amplified polymorphic DNA (RAPD) markers meet these general requirements, but are prone to certain types of error that affect reproducibility (Davin-Regli et al., 1995; Hansen et al., 1998; Perez et al., 1998; Staub et al., 1996). These reproducibility problems limit interlaboratory method transferability, and make routine scoring and comparison to an existing database difficult. In addition, most bands (markers) generated using RAPD by any one primer are not polymorphic. This necessitates execution of multiple polymerase chain reactions (PCR) with different primers for adequate fingerprinting of each sample.

Received for publication 21 Aug. 2001. Accepted for publication 8 Apr. 2002.

${ }^{1}$ Assistant research director and corresponding author.

${ }^{2}$ Professor.
Accurate computer automation would facilitate scoring, cataloging, and comparisons of cranberry DNA fingerprinting data. However, some of the shortcomings of RAPD markers make the implementation of computer-based analysis problematic. In this case, reproducibility must be increased, markers must be unambiguous, and monomorphic markers must be minimized.

Sequence characterized amplified regions (SCARs) which are derived from RAPD markers, have the advantages of RAPD markers and the additional benefits of increased specificity and reproducibility (Paran and Michelmore, 1993). SCAR primer sets can be strategically multiplexed in a PCR reaction to improve efficiency (i.e., increase the number of polymorphic markers produced per reaction). Thus, it is conceivable that the number of reactions required to fingerprint one sample could be reduced to one.

The most significant drawback of SCAR markers is development time and expense. Once established, however, the cost of individual SCAR PCR reactions are similar to those of RAPD markers, and a reduction in the number of required reactions can decrease overall expenses. Additionally, SCAR markers lend themselves readily to computer automation (i.e. scoring, database cataloging, and comparative analysis). The advantages of SCAR markers over RAPD markers for large-scale and ongoing DNA fingerprinting of cranberry samples could easily justify the added development effort. Therefore, the objectives of this research were to 1) develop a fast, inexpensive and reliable method for DNA fingerprinting of American cranberry, 2) evaluate the efficiency of the SCAR marker and RAPD marker methods for discriminatory analysis, 3) compare estimates of genetic relatedness among cranberry germplasm based on RAPD and SCAR marker derived data, and 4) assess application of the SCAR methodology for computer automation using fluorescent dye-labeled primers and laser detection of products.

\section{Materials and Methods}

Plant material, DNa extraction, and Quantification. Cultivars used were deduced previously to be true-to-type by examina- 
Table 1. Cultivars used in this study.

\begin{tabular}{|c|c|c|c|c|}
\hline Cultivar $^{2}$ & $\mathrm{Ab}^{\mathrm{y}}$ & Parentage & Origin & Released $^{\mathrm{x}}$ \\
\hline Ben Lear & $\mathrm{BL}$ & Wild selection & Berlin, Wis. & 1901 \\
\hline Early Black & $\mathrm{EB}$ & Wild selection & Harwich, Mass. & 1852 \\
\hline Franklin & FR & Hybrid, EB x HO & Whitesbog, N.J. & 1961 \\
\hline Howes & $\mathrm{HO}$ & Wild selection & East Dennis, Mass. & 1843 \\
\hline LeMunyon & LM & $\mathrm{NA}^{\mathrm{w}}$ & NA & NA \\
\hline McFarlin & MF & Wild selection & South Carver, Mass. & 1874 \\
\hline Pilgrim & PI & Hybrid, Prolific x MF & Whitesbog, N.J. & 1961 \\
\hline Stevens & ST & Hybrid, MF x Potter & Beltsville, Md. & 1950 \\
\hline Wilcox & WI & Hybrid, HO x Searles & Beltsville, Md. & 1950 \\
\hline
\end{tabular}

${ }^{\mathrm{z} C u l t i v a r}$ information source Eck (1990).

${ }^{y}$ Abbreviation for cultivar name used throughout this paper.

${ }^{\mathrm{x}}$ Year cultivar was released under its current name.

${ }^{\mathrm{w}}$ Information not available.

tion of multiple accessions from several sources throughout North America via RAPD analysis, and comparison of morphological and phenological traits as described in Eck (1990). The cultivars used were 'Ben Lear', 'Early Black', 'Franklin', 'Howes', 'McFarlin', 'Pilgrim', 'LeMunyon', 'Stevens', and 'Wilcox' (Table 1). Various researchers collected the control cultivars and germplasm accessions representing putative cultivars (Table 2) between 1988 and 1993 from growing regions in New Jersey, Massachusetts, Wisconsin, Oregon, Washington, and British Columbia. All of the accessions are maintained in genetically homogeneous field plots at the Philip E. Marucci Center for Blueberry and Cranberry Research and Extension, Rutgers University, Chatsworth, N.J. 'Early Black' and 'Stevens' stock plants were self-pollinated and the first generation (S1) progeny from each were maintained in square 0.95 -L pots under standard greenhouse conditions. Once developed, the SCAR marker system was utilized to fingerprint $>500$ additional cranberry accessions. Details regarding the origin of these accessions are available upon request.

DNA was isolated from four to five fresh, fully expanded leaves $(\approx 30 \mathrm{mg})$ using the method described by Stewart and Via (1993) as modified by Novy and Vorsa (1995). Isolated DNA for amplification was quantified using a fluorometer (DYNA Quant 200, Amersham Pharmacia Biotech, Piscataway, N.J.).

RAPD MARKER CONVERSIONS TO SCAR MARKERS. RAPD reactions using DNA isolated from nine cranberry cultivars (Table 1), and their analysis including polyacrylamide gel electrophoresis and silver staining for marker detection were as described by Novy et al. (1994). Thirty-three 10-mer primers were screened and selected for amplification of distinct, reproducible polymorphic markers based on previous experience with RAPD analysis of cranberry (Novy and Vorsa, 1995; Novy et al., 1994; Polashock and Vorsa, unpublished). Those tested included selections from Operon Technologies (Alameda, Calif.) sets OPA, OPB, and OPC, as well as selections from the University of British Columbia Biotechnology Laboratory (Vancouver, British Columbia, Canada) sets 4 and 5. Silver staining was used for marker detection in the initial screens since it is much more sensitive than ethidium bromide $(\mathrm{EtBr})$ staining and provides for a permanent record (gels were dried between two sheets of cellulose film for archiving). However, since the silver staining process fixes the DNA in the gel, samples with bands to be excised and the DNA isolated were rerun and the gels stained with EtBr. To avoid overlap in marker sizes, enhance ease of interpretation, and minimize confounding markers in planned multiplex reactions, RAPD bands selected for SCAR conversion were chosen to span the size range from $\approx 300$ to 1700 base pairs (bp) with adequate separation (at least $50 \mathrm{bp}$ ) between the bands. Initially, 10 bands were selected for extraction, sequencing, and SCAR primer design. The selected bands were excised from the EtBr stained gel(s) and the DNA was recovered by incubating the gel slice in two volumes of $0.5 \mathrm{M}$ ammonium acetate/1.0 mM ethylenediaminetetraacetic acid (EDTA) at $37{ }^{\circ} \mathrm{C}$ overnight, followed by precipitation with two volumes of $95 \%$ ethanol (Ausubel et al., 1995). The purified fragments were cloned directly into the pGemT vector (Promega Corp., Madison, Wis.) following the manufacturers protocol. All plasmids were propagated in E. coli strain DH5 $\alpha$ (LifeTechnologies, Rockville, Md.). Plasmids were isolated and column purified using a commercial kit (MOBIOLaboratories, Solana Beach, Calif.). The ends of the cloned fragments were sequenced using Cy5 labeled M13 forward and reverse primers and the SequiTherm EXCEL II Long-Read kit for the ALF (Epicentre, Madison, Wis.). Reaction products were separated and detected on an ALF Express sequencer (Amersham Pharmacia Biotech) and the resulting sequences were analyzed using Lasergene software (DNASTAR, Inc., Madison, Wis.). Primers designed to amplify SCAR markers included the sequence of the original 10-mer primer and an additional 11 to 15 bases 3 ' to the 10-mer (Paran and Michelmore, 1993). Both forward and reverse primers were designed for each cloned fragment.

Primer Testing. Newly designed SCAR primer pairs were tested in $13 \mu \mathrm{L}$ PCR reactions containing $1 \times$ Stoffel buffer, $2.5 \mathrm{~mm}$ $\mathrm{MgCl}_{2}, 0.2 \mathrm{~mm} \mathrm{dNTPs}, \approx 0.38 \mu \mathrm{M}$ of each primer, and 1.25 units of

Table 2. Control cultivars and germplasm accessions used for comparison of the RAPD and SCAR marker systems.

\begin{tabular}{ll}
\hline \hline Accession designation $^{\mathrm{z}}$ & Cultivar $^{\mathrm{y}, \mathrm{x}}$ \\
\hline FR & Franklin (control) \\
MF & McFarlin (control) \\
PI & Pilgrim (control) \\
ST & Stevens (control) \\
WI & Wilcox (control) \\
\#35 & \#35 (control) \\
US88-21, US89-2, US94-155 & Beckwith \\
US88-25, US88-93, US89-4 & Pilgrim \\
US88-32, US89-9 & Stevens \\
US88-34 & Wilcox \\
US88-47, US89-10, US94-10 & Crowley \\
US88-51 & \#35 \\
US88-58, US89-1, US94-11, US94-40, US94-1466 & Bergman \\
US89-6, US89-8 & McFarlin \\
US94-13 & Franklin
\end{tabular}

$\overline{{ }^{\mathrm{z}} \text { Abbreviation (for controls) or accession number assigned to the clone }}$ when collected.

${ }^{y}$ Actual cultivar (for controls) or putative cultivar at the time of collection.

${ }^{x}$ Note that controls were confirmed previously to be true-to-type as described in the text, while cultivar identity has not been confirmed for all other accessions. 
Table 3. SCAR primers developed for cranberry cultivar discrimination.

\begin{tabular}{llc}
\hline \hline $\begin{array}{l}\text { Primer } \\
\text { no }^{z}\end{array}$ & $\begin{array}{l}\text { Primer } \\
\text { sequence }\end{array}$ & $\begin{array}{c}\text { Multiplex } \\
\text { mix }^{\mathrm{y}}\end{array}$ \\
\hline 5 & CCG CAT CTA CAT AAT CGA ATC G & 1 \\
6 & CCG CAT CTA CAA ATA CAA AAA TG & 1 \\
9 & GTT TCG CTC CCG TGG AGG TAC T & 1 \\
10 & GTT TCG CTC CTC TTA TCA ATG T & 1 \\
21 & CAA TCG CCG TAT CGG TTA GAG & 2 \\
22 & CAA TCG CCG TCA ATT TCT CAG & 2 \\
25 & GTT TCG CTC CAC AAT TTT CTT AG & 1 \\
26 & GTT TCG CTC CTC TGC AAA TCA TTT G & 1 \\
48 & TTG CGT CAT GTA TTG TGA AAA AAA & 1 \\
49 & TTG CGT CAT GAT GAG ATT TGA ATA & 1 \\
50 & AGG CGG GAA CGT AGT CGG CTT AGG & 1 \\
51 & AGG CGG GAA CCA AAT TAC ATA TAT & 1 \\
56 & GAA TGG TGA GAT TCA GAA CTT GTG & 2 \\
57 & GAA TGG TGA GCA TAA TGA GTC CTG & 2 \\
58 & GGG TGG ACA TTT TTT CAA TTT GTG & 2 \\
59 & GGG TGG ACA TGT GAC CAA GAA AG & 2 \\
64 & GGA TCT ATG CAT AAT TAA CGA ACA & 2 \\
65 & GGA TCT ATG CTT CTG TAT TGT ATT & 2
\end{tabular}

${ }^{\mathrm{z}}$ Number as assigned to each primer during the design procedure. Primers are listed in pairs.

${ }^{\mathrm{y}}$ Multiplex mix to which primer was added (e.g., primers 5 and 6 are a single primer set used in multiplex reaction number 1 ).

Stoffel fragment (PE Applied Biosystems, Foster City, Calif.). All other reaction parameters were as those used for RAPD markers except that the annealing temperature was raised from 36 to $59^{\circ} \mathrm{C}$. Resulting products were separated on polyacrylamide gels and silver stained. Primer sets that amplified the desired polymorphic marker were retained for use in multiplex PCR reactions. If any SCAR primer set failed to produce the desired band, a different RAPD marker, similar in size to the one that failed, was extracted, cloned, and sequenced. A new primer set was then designed to amplify the replacement band(s), and tested as stated above. Multiplex reactions were performed as single pair reactions, except that several primer pairs were added to each reaction. The volume of the multiplex reactions was later increased to $26 \mu \mathrm{L}$ to accommodate the addition of 8 to 10 primers. The final concentrations of all components in the $26 \mu \mathrm{L}$ reactions were the same as those in the $13 \mu \mathrm{L}$ reactions except for the primers, which were reduced to $0.19 \mu \mathrm{M}$ each. Two multiplex PCR reactions were developed to distinguish the major cranberry cultivars (Table 1 ). The primers are detailed in Table 3.

SCORING AND Cluster ANALYSIS. Markers (both RAPD and SCAR) were scored for presence (1) or absence (0) and the data were analyzed using NTSYS-pc (Exeter Software, Setauket, N.Y.). Similarity was estimated using SIMQUAL (similarity for qualitative data) and the DICE (Dice, 1945) coefficient, and clustering was performed using unweighted pair-group method arithmetic average (UPGMA). The phenogram was generated from the cluster analysis using tree display (TREE).

Correlation anALYSIS. All of the newly developed SCAR markers were tested for correlation with each other using Pearson's coefficients with the PROC CORR program of SAS (SAS Institute Inc., Cary, N.C.).

EFFICIENCY OF RAPD AND SCAR MARKERS. To compare the separation power as well as the genetic similarity inferences of SCAR versus RAPD markers, a subset of cranberry accessions (see Table 2) was assayed using both techniques. SCAR markers were generated using two multiplex reactions, together containing a total of nine primer sets (Table 3). The two multiplex SCAR reactions were predicted to yield many more useful markers than a typical RAPD reaction. Therefore, to obtain a similar number of markers for cluster analysis, RAPD markers were generated using four separate PCR reactions. Cluster analysis was performed using the resultant data as described above.

SEPARATION OF SELF-PROGENY. To test the discrimination efficiency of SCAR markers among closely related individuals, S1 progeny from 'Stevens' $(n=51)$ and 'Early Black' $(n=41)$ were DNA fingerprinted and scored using SCAR markers. Cluster analysis of these data was performed as described above. While 'Stevens' was chosen to represent a relatively recently released cultivar (1950), 'Early Black' represents a wild selection first described in 1852 (Eck, 1990). Both 'Stevens' and 'Early Black' are widely cultivated in major cranberry growing regions.

FLUORESCENT LABELING AND DETECTION. To adapt SCAR primers for laser detection, one primer from each primer set was labeled with the fluorescent dye Cy5 (labeled primers were purchased from Integrated DNA Technologies, Coralville, Iowa). The labeled multiplex reactions were run as for the unlabeled reactions except that the labeled reactions were also tested using a hot-start Taq polymerase (Platinum Taq, Life Technologies Inc., Rockville, Md.) instead of Stoffel fragment. The buffer used in reactions with the Platinum Taq was that provided by the manufacturer (Life Technologies Inc.) instead of Stoffel buffer. All other conditions were as used for the Stoffel fragment-containing reactions. Products were separated and detected on an ALF Express sequencer (Amersham Pharmacia Biotech) using $6.75 \%$ (nondenaturing) polyacrylamide gels and $0.5 \times$ TBE running buffer in a short plate format $(14 \mathrm{~cm}$, with $0.5 \mathrm{~mm}$ spacers). Run conditions were $400 \mathrm{~min}, 800 \mathrm{~V}, 60 \mathrm{ma}, 15$ $\mathrm{W}$, at $25^{\circ} \mathrm{C}$ with a 2-s sampling interval. Results were analyzed using Fragment ManagerV1.2 (AmershamPharmaciaBiotech) and the sizes of the labeled products were estimated by comparison to Cy5 50 to 500 bp size marker (Amersham Pharmacia Biotech). Aliquots of fluorescent reactions were also separated on mini polyacrylamide gels (as for RAPD reactions) and then silver stained for direct comparison to unlabeled reaction products.

REPRODUCIBILITY AND INTERLABORATORY TRANSFERABILITY. TO assess reproducibility and the potential for interlaboratory transferability, the SCAR marker system was tested in another laboratory. Nine cultivars (Table 1) were tested in two multiplex PCR reactions (for primers see Table 2). The thermocycler used was an Uno Thermoblock (Biometra, Gottingen, Germany). Reactions were performed in $0.5 \mathrm{~mL}$ thin wall tubes (Lab Scientific, Livingston, N.J.), using Taq polymerase (Life Technologies, Inc). Run conditions were exactly as described above for SCAR analysis.

\section{Results}

Of the 33 RAPD primers screened, $18(\approx 55 \%)$ yielded polymorphic bands suitable for further testing. Among those 18 primers, $\approx 50$ bands (fragments) were polymorphic among the cultivars tested (Table 4). There was, however, significant overlap in the sizes of the polymorphic fragments. For example, six bands were in the range of 500 to $550 \mathrm{bp}$ and seven were in the range of 600 to $670 \mathrm{bp}$. Initially, 10 bands that spanned the size range from about 300 to $1700 \mathrm{bp}$ were cloned, sequenced, and used for SCAR primer design.

Not all SCAR primer sets functioned as desired. There were three main types of failure to produce the desired bands. The first type (Type 1) was the loss of polymorphism (i.e., the same band was amplified in all or most cultivars tested). The second type (Type 2), 
Table 4. RAPD primers that provided reliable polymorphic bands in cranberry.

\begin{tabular}{ll}
\hline \hline Primer $^{2}$ & $\begin{array}{l}\text { Polymorphic band designation } \\
\text { (size in base pairs) }\end{array}$ \\
\hline OPA-9 & $600,800,880$ \\
OPA-11 & $520,730,750,800,1400$ \\
OPA-12 & $600,650,670,710,770$ \\
OPA-17 & $650,1100,1400$ \\
OPB-1 & 800,1000 \\
OPB-7 & 540,1075 \\
OPB-8 & $400,500,550$ \\
OPC-2 & 1400,1700 \\
OPC-4 & 360 \\
OPC-6 & 300,700 \\
OPC-8 & $400,500,550$ \\
OPC-9 & 1100 \\
OPC-11 & 400,700 \\
BC402 & 1500,1550 \\
BC471 & $600,700,770,800,1300$ \\
BC472 & $430,490,550$ \\
BC474 & $580,660,1050,1400$ \\
BC500 & 1100,1150
\end{tabular}

${ }^{\mathrm{z}} \mathrm{OP}=$ Operon Technologies, Alameda, Calif., $\mathrm{BC}=$ University of British Columbia Biotechnology Laboratory, Vancouver, British Columbia, Canada.

was the amplification of multiple bands of similar or varied sizes, or a smear of products instead of a distinct single band. The third type (Type 3) was where no bands were amplified. Those primers that consistently amplified at least one distinct polymorphic marker band were evaluated in multiplex reactions.

Some primer sets that performed well individually did not appear to function in multiplex reactions and were eliminated from further testing. In which case, a different polymorphic RAPD band was selected and new SCAR primers were designed and tested as described above. This process was continued until a sufficient number of SCAR primers were developed to reliably separate the nine commonly grown cranberry cultivars used in this study. This required the cloning, primer design, and testing of 25 additional bands, for a total of 35 (including the original 10 bands that were selected). Success rate from isolated band to useful multiplex SCAR primer set was about $25 \%$ ( 9 primer sets of 35). The most common failure was Type 1 (15 of 35), followed by Type 2 ( 8 of 35), and finally, Type 3 (3 of 35).

Although a single multiplex reaction containing all SCAR primers was desired, PCR reactions containing more than five primer sets (i.e., 10 primers) did not perform adequately using the volumes and conditions described above. Thus, two multiplex reactions were developed with four and five primer sets to adequately separate the cultivars tested (Table 3 ). A typical RAPD reaction yielded one to five polymorphic bands (Fig.1A), while the multiplex SCAR system exhibited 14 polymorphic bands for primer mix1 (Table 5, Fig. 1B) and 13 polymorphic bands for primer mix 2 (gel not shown, for markers scored see Table 5). Note that the SCAR marker reactions are easier to score, and appear to be more reliable than the RAPD marker reactions (the RAPD reaction for cultivar ' $\mathrm{LM}$ ' did not work well and cultivar \#35 is light).

Since the total number of scored markers (27) exceeded the number of primer sets (9), some primers sets yielded more than one marker, in which case the occurrence of some markers might be correlated. When all of the markers were tested for mutual relation, five markers were found to be fairly highly correlated $(r>0.75)$, with four of the five marker pairs having a correlation of $r \geq 0.94$. These five correlations were all positive (i.e., when one marker was present, the other tended to be present). The highest negative correlation was $r=-0.42$ and was not significant.

SCAR markers were compared to the RAPD markers for genetic discrimination using 27 cranberry accessions (Table 2). Since the SCAR markers were derived from RAPD markers, it would be expected that, using the same number of markers, the discrimination efficiency and the resulting phenogram would be very similar between the two marker systems. RAPD marker analysis required use of four separate 10-mer primer reactions to approximate the same number of markers derived from the two multiplex SCAR marker reactions for this particular set of clones. Both marker systems allowed separation of most accessions, and although the phenograms were not identical, the inferred relationships of most of the accessions were similar (Fig. 2). However, some differences were expected since the markers used were not identical. At or above a value of 0.9 coefficient of similarity (i.e., the most closely related clones), groupings were generally maintained between the two systems. Even at this level of genetic similarity, however, differences were apparent. For example, 'US88-47' and 'US89-2' were not separated using RAPD markers, while with the SCAR marker system similarity between these two clones was about 0.68 . Similarly, SCAR marker analysis discriminated 'US89-4' from 'Pilgrim' whereas with RAPD markers, these clones were not distinguishable. Conversely, RAPD analysis discriminated 'US89-9' from 'Stevens' while these clones were not separated by SCAR analysis. Inferred genetic relationships with similarity below 0.9 were not consistent between these marker systems.

The efficacy of the SCAR marker system discrimination efficiency of first generation self progeny was examined using progeny derived from self-pollination of the cultivars Early Black and Stevens. SCAR markers, although cultivar-dependent, separated almost all $S 1$ progeny tested from the parent. Only one 'Early Black' $\mathrm{S} 1$ individual $(\approx 3 \%)$ could not be distinguished from the parent, while five 'Stevens' S1 progeny $(\approx 10 \%)$ could not be distinguished from the parent (Fig. 3).

Fig. 1. Typical results of cranberry cultivars analyzed using a (A) RAPD primer OPB-7 and (B) SCAR multiplex primer mix 1. Cultivar abbreviations are as detailed in Table 1 with the exception of $\mathrm{B} 1$ and $\mathrm{B} 2$, which are two different accessions of 'Bergman' ('Early Black' x 'Searles') which was released in 1961 from Whitesbog, N.J. M $=100 \mathrm{bp}$ molecular weight ladder (Life Technolgies, Rockville, Md.). Marker sizes are in $100 \mathrm{bp}$ increments with the exception of the highest marker (2000 bp). Some markers are labeled for reference.
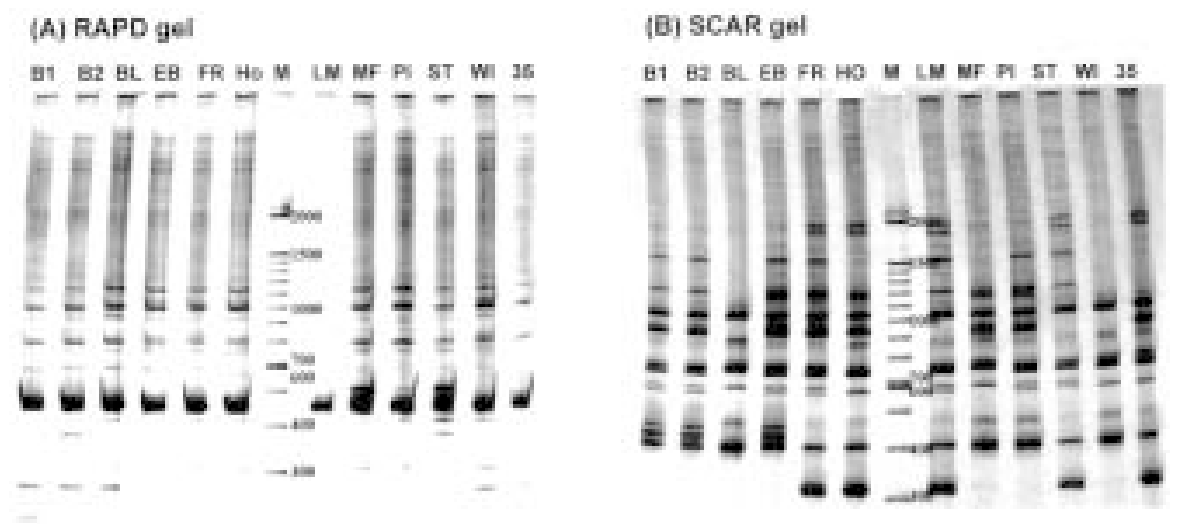
Table 5. SCAR markers generated and scored using two multiplex PCR reactions.

\begin{tabular}{|c|c|c|c|c|c|c|c|c|c|}
\hline Markers ${ }^{\mathrm{y}}$ & \multicolumn{9}{|c|}{ Cultivar $^{\mathrm{Z}}$} \\
\hline $1 / 320$ & $0^{x}$ & 0 & 1 & 1 & 0 & 1 & 0 & 1 & 0 \\
\hline $1 / 440$ & 0 & 1 & 0 & 0 & 0 & 0 & 0 & 0 & 0 \\
\hline $1 / 460$ & 0 & 1 & 0 & 0 & 0 & 0 & 0 & 0 & 0 \\
\hline $1 / 590$ & 0 & 1 & 1 & 1 & 0 & 1 & 1 & 1 & 1 \\
\hline $1 / 750$ & 1 & 1 & 1 & 0 & 1 & 1 & 1 & 1 & 0 \\
\hline $1 / 900$ & 0 & 1 & 1 & 1 & 1 & 0 & 1 & 0 & 0 \\
\hline $1 / 1150$ & 0 & 1 & 1 & 1 & 1 & 1 & 1 & 1 & 0 \\
\hline $1 / 1200$ & 0 & 1 & 1 & 1 & 1 & 0 & 1 & 0 & 0 \\
\hline $1 / 1500$ & 0 & 1 & 1 & 0 & 0 & 1 & 1 & 1 & 0 \\
\hline $1 / 1800$ & 0 & 0 & 1 & 1 & 0 & 1 & 0 & 1 & 0 \\
\hline $2 / 465$ & 1 & 0 & 1 & 1 & 0 & 0 & 0 & 0 & 1 \\
\hline $2 / 475$ & 1 & 1 & 1 & 0 & 1 & 1 & 1 & 1 & 1 \\
\hline $2 / 550$ & 0 & 1 & 1 & 1 & 1 & 1 & 1 & 1 & 1 \\
\hline $2 / 600$ & 1 & 0 & 0 & 1 & 0 & 0 & 1 & 0 & 1 \\
\hline 2/980 & 1 & 1 & 1 & 1 & 0 & 0 & 1 & 0 & 1 \\
\hline $2 / 1000$ & 1 & 0 & 1 & 0 & 0 & 0 & 0 & 0 & 1 \\
\hline $2 / 1100$ & 0 & 1 & 0 & 0 & 0 & 0 & 1 & 1 & 0 \\
\hline $2 / 1150$ & 0 & 0 & 1 & 1 & 0 & 0 & 0 & 0 & 1 \\
\hline $2 / 1200$ & 0 & 0 & 1 & 1 & 0 & 0 & 0 & 0 & 1 \\
\hline $2 / 1500$ & 0 & 1 & 0 & 0 & 0 & 0 & 1 & 1 & 0 \\
\hline $2 / 1750$ & 0 & 0 & 0 & 1 & 0 & 0 & 1 & 0 & 1 \\
\hline
\end{tabular}

${ }^{\bar{z}}$ Cultivar abbreviations as described in Table 1.

${ }^{y}$ Number corresponds to the SCAR multiplex primer mix number/marker size in base pairs.

${ }^{\mathrm{x}} 1=$ presence of marker, $0=$ absense of marker.

Side by side reaction comparisons using unlabeled primers and Cy5 labeled primers for fluorescent detection indicated some loss of product when Cy5 is used. When the reactions were separated on polyacrylamide gels and visualized with silver staining, product reduction using Cy5 labeled primers was evident as a marked decrease in band intensity (Fig. 4). This effect was somewhat primer set specific. Depending on the multiplex mix used, some predicted markers were not clearly visible. Such losses were generally greater for the higher molecular weight fragments. During ALF Express analysis, fluorescent SCAR markers appear as peaks (Fig. 5). Most of the polymorphisms seen on the silver stained gel (Fig 1B) were evident in the ALFexpress output (Fig. 5). However, one problem was lack of separation of some peaks of very similar molecular weight. Note for example the four lowest molecular weight (400 to $450 \mathrm{bp}$ ) bands in samples B1 and B2 (Fig. 1B). These appear as one large peak, with a slight separation, at $\approx 500$ bp in the chromatogram shown in Fig. 5. Thus, all four bands distinguishable on the silverstained gel are not visible in the chromatogram.

To get an idea of reliability and interlab transferability, the SCAR marker system, using DNA samples from our laboratory, was tested at another laboratory using a different thermocycler, different Taq polymerase, and different reaction tubes. Results were identical to those obtained in our laboratory (data not presented).

\section{Discussion}

The success of SCAR primer set development was unpredict- able, necessitating repeated testing. Type 1 failure (loss of polymorphism) suggests that specificity was associated with the original 10mer primer binding region. When converted to SCAR primers, the increased length presumably allowed better annealing of the primer during PCR resulting in amplification across most samples. The other extreme, Type 3 failure (no amplification), suggests that one or more of the primers in that particular primer set were not functional under the desired PCR conditions of the SCAR reactions. Annealing temperature was likely a factor in such failures. Annealing temperature was raised from $36^{\circ} \mathrm{C}$ in RAPD to $59^{\circ} \mathrm{C}$ for $\mathrm{SCAR}$ reactions. Nevertheless, other parameters such as magnesium concentration and primer characteristics such as hairpin formation could also be important factors. Some of the primer sets that failed could probably be utilized by fine adjustment of the PCR parameters. Since ideal primers could not be designed, due to the restriction of using the original 10-mer RAPD primer region and the sequences immediately adjacent to that region, it is not surprising that some primers were operatively problematic. The amplification of multiple bands (Type 2 failure) could be due to amplification of variable repeat regions or alternate alleles. Unstable primer binding occurring under the selected conditions could have given rise to the smearing observed in the products of some reactions. Although some SCAR primer sets amplified more than one distinct band, these primer sets were still used if one or more of the amplified bands were polymorphic.

Paran and Michelmore (1993) found that six out of nine RAPD polymorphisms were caused by one or more mismatches in the 


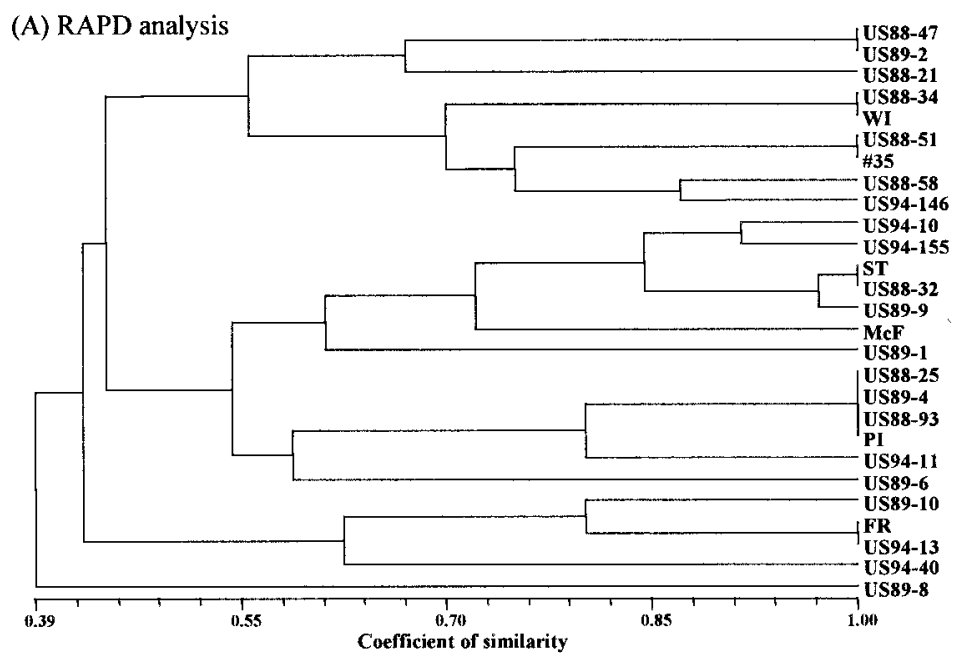

(B) SCAR analysis

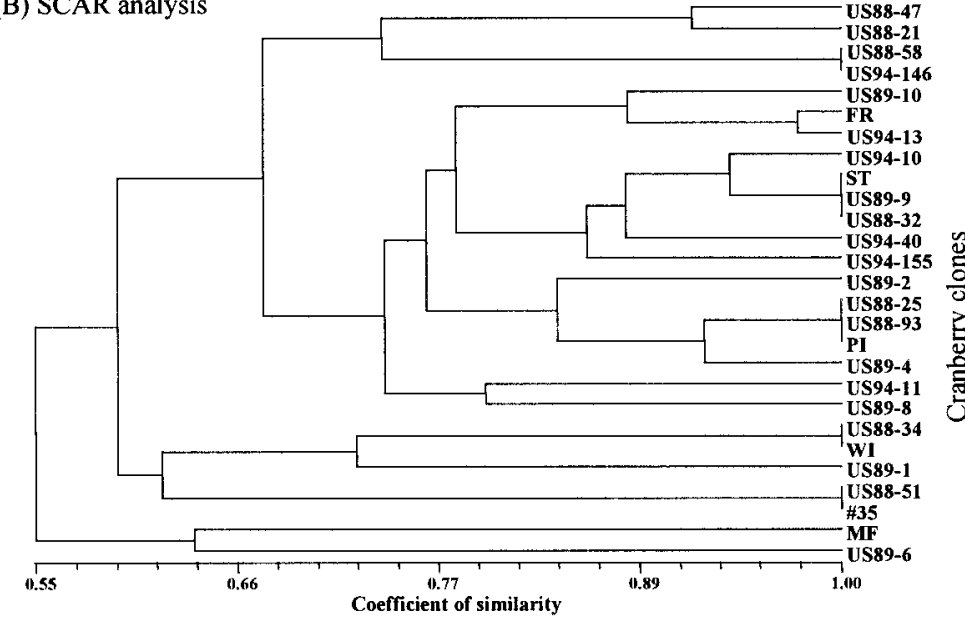

Fig. 2. An UPGMA phenogram derived from (A) RAPD and (B) SCAR analysis on a subset of cranberry clones. Cultivars are as detailed in Table 2.

priming region. This could cause loss of polymorphism (Type 1 failure) after conversion to SCAR markers. Paran and Michelmore (1993) also described instances where more than one marker (of a different size) was produced from a single SCAR primer set. These markers were described as potentially codominant. Amplification of several closely sized bands (Type 3 failure) was described for raspberry (Rubus idaeus L. and $R$. idaeus $\times$ R. occidentalis L.) (Parent and Page, 1998) and strawberry (Fragaria L. sp.) SCAR markers (Haymes et al., 2000). Horejsi et al. (1999) reported that only $15 \%$ of 48 SCAR primer pairs developed produced the original RAPD single band polymorphisms in cucumber (Cucumis sativus L.). The authors further noted that $42 \%$ of the 48 SCAR primer pairs developed produced multiple bands.

Several SCAR primer sets that worked well alone did not perform well in multiplex reactions. Multiplex reactions require that the primer sets function at similar annealing temperatures, not interact with each other, and have similar reaction condition optima (magnesium concentration, etc.). Thus, two multiplex reactions were developed that included either four or five primer sets that adequately and reliably separated the cultivars examined. Problems associated with multiplexing have been described by several groups
(Amicucci et al., 2000; Henegariu et al., 1997; Parent and Page, 1998). Multiplexing, however, was considered to be critical to our purposes since one goal was to reduce the number of reactions required which would adequately discriminate common cranberry cultivars.

Although both the RAPD and SCAR systems were effective for genotypic discrimination, inferences of genetic similarity were not consistent between these marker systems when the similarity coefficient was below 0.9 . Inferences of genetic similarity based on DNA fingerprinting must be made with caution (Lynch, 1988).

Accessions with identical SCAR marker fingerprints are likely closely related or identical clones. The fact that many of these accessions were collected originally as representatives of the same named cultivar supports this hypothesis. For example, accessions 'US88-25', 'US88-93' and the cultivar Pilgrim had identical SCAR marker fingerprints (Fig. 2B). These 'US' accessions were collected in 1988 and 1989 as representatives of the cultivar Pilgrim suggesting that all of the accessions are the same genotype. However, since self-pollination could give rise to individuals with fingerprints identical to the parent, this leaves open the possibility that some of the identical accessions represent S1 progeny. Using a larger number of independent markers should provide a statistical measure to evaluate this hypothesis.

SCAR fingerprinting of additional accessions, from a germplasm collection, allowed recovery of additional size polymorphisms (data not presented). However, since the new markers were amplified in multiplex reactions, they could not be attributed to a particular primer set. Using both SCAR primer mixes ( 9 primer sets), 38 markers were routinely scored across the germplasm (594 accessions) analyzed thus far. Analysis of all SCAR markers used in this study revealed that some were positively correlated. Correlation between some of the markers might be expected since only nine primer pairs were used to score a total of 38 markers and there are only 12 linkage groups in cranberry. Of the six highest correlations noted out of 703 pairwise comparisons, only four were over $r$ $=0.9$. One of each set of the most highly correlated markers could probably be eliminated from the analysis with little effect on the overall results. This would remove only two of the 38 markers used in this study. One hypothesis to explain how high correlations could arise is that more than marker could be amplified from the same locus.

The number of SCAR markers required to achieve a reasonable degree of certainty in detecting genetic differences is dependent on several factors. These include the amount of genetic variation in the population, the degree of polymorphism of the particular markers used, and inheritance associated with each marker. Correlation between the markers must also be taken into account when calculating the number of markers required. Assuming that all selected markers are heterozygous, unlinked, and segregate normally (Mendelian), 10 markers would be expected to differentiate selfed progeny from the parent $\approx 95 \%(0.75)^{10}$ of the time (i.e., $\approx 5 \%$ would be expected to have the same pattern as the parent). Using 15 markers, expected differentiation increases to $\approx 99 \%(0.75)^{15}$. Therefore, to analyze progeny from selfing, 10 to 20 markers should be sufficient to differentiate most progeny from the parent with fairly high certainty. Progeny from intercrosses of cultivars should be even easier to differentiate from the parents since more loci and more alleles per locus would be expected to be segregating.

The newly developed SCAR marker system worked well for 


\section{(A) Early Black derived S1 progeny}

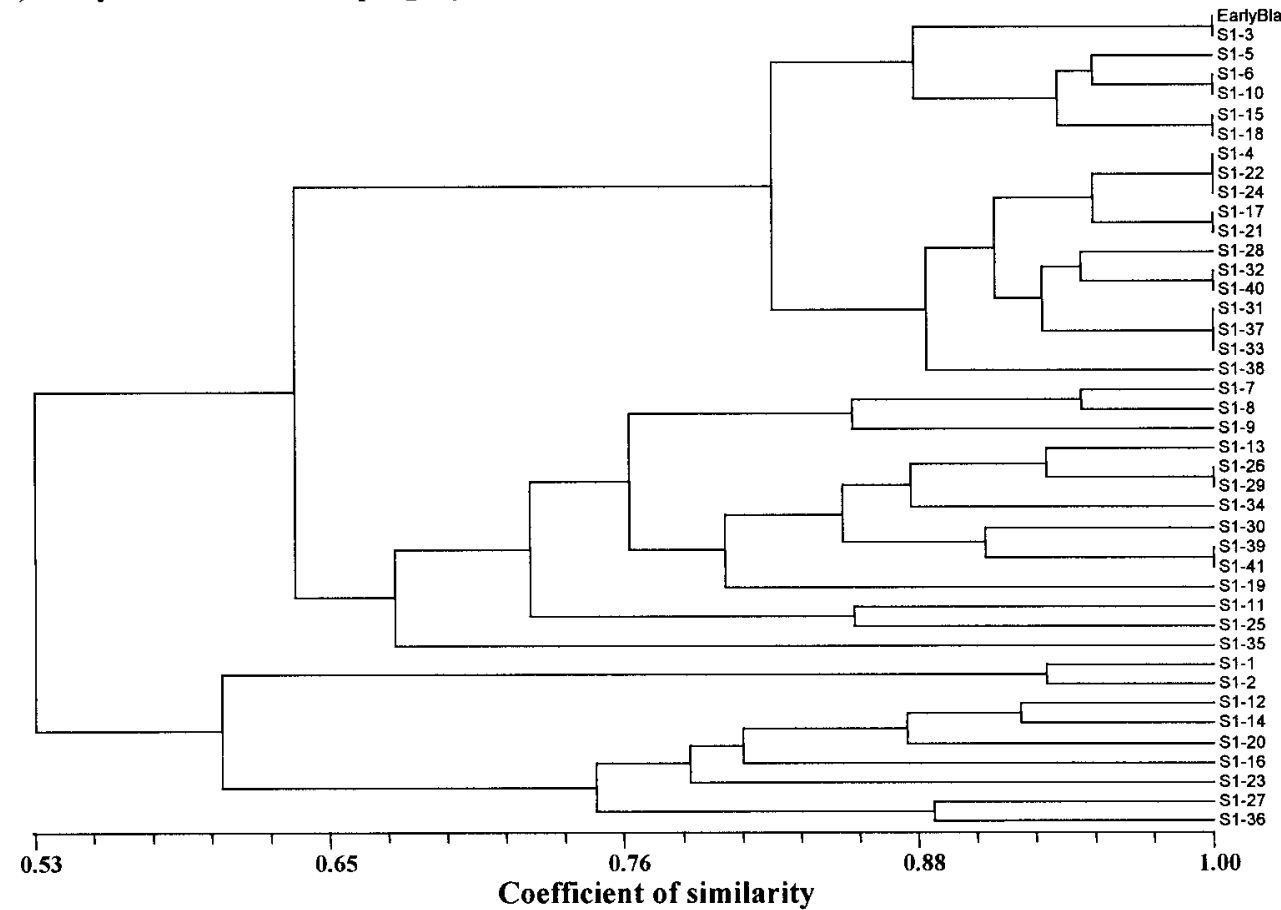

Coefficient of similarity

(B) Stevens and derived S1 progeny

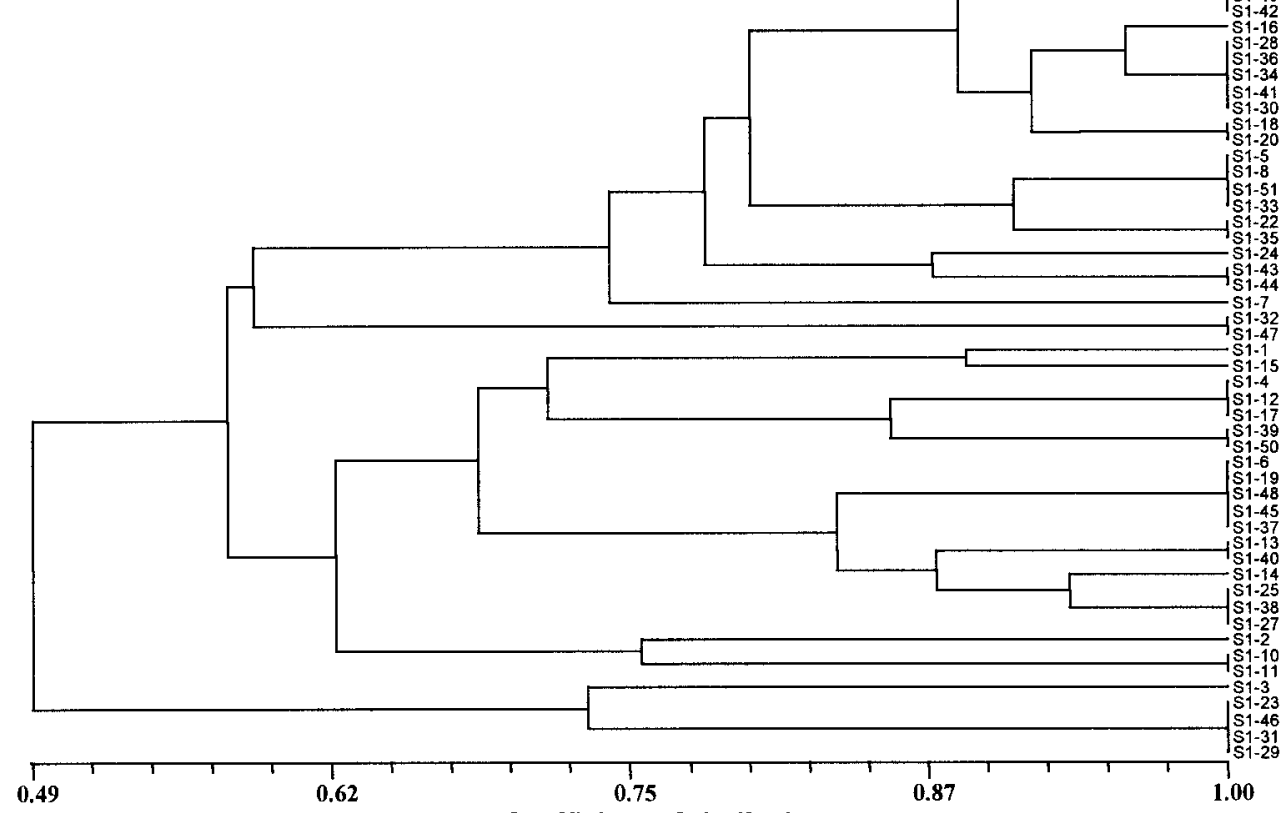

Coefficient of similarity

Fig. 3. An UPGMA phenogram derived from SCAR analysis of self (S1) progeny of (A)'Early Black’ and (B) 'Stevens'.

cultivar discrimination, but it was also important to distinguish the cultivars from closely related off-types, particularly those arising from self-pollination. Self-progeny would most likely be the most common source of off-types in cranberry beds since cranberry is extremely self-fertile (Sarracino and Vorsa, 1991). Novy and Vorsa (1995) suggested that the RAPD markers used in their study might not have been sufficient to distinguish between the parent cultivar ('Howes') and self-progeny. In that study, 66 markers, derived from reactions using 22 primers, were used. Here we have shown that with only two multiplex reactions, we can separate most of the self-progeny from the two cultivars tested. AdditionalSCAR marker primers could be employed to further reduce the chance of mis-identifying self-progeny as the parent.

Use of fluorescently labeled reactions would greatly enhance automation of fingerprinting, since the products of the reactions could be separated, and more importantly detected and recorded, using an apparatus such as a fluorescent sequencer. This system was used successfully for microsatellite analysis (Moscetti et al., 1995). Automatic detection eliminates the separate step of silver staining and allows for nonbiased recording of the results. In addition, more samples could be run on a single gel. We tested the potential of using this system by incorporating Cy5 fluorescent dye into the SCAR primers. Although the dye used was specific for detection on the AlfExpress automated sequencer (Amersham Pharmacia Biotech), this approach could be extended to include any fluorescent dye coupled with a separation/detection system from another manufacturer.

The conversion of the SCAR marker multiplex system to fluorescent detection using Cy5 labeled primers was successful. Some potential problems such as a loss in product quantity were evident. This reduction in quantity could have been due to contaminants in the labeled primer mixes. However, since the primers were highly purified (by high-performance liquid chromatography), the more likely reason is inefficient primer binding to the genomic DNA due to the presence of the large dye molecule. This problem was alleviated partially using a hot-start type of Taq polymerase (Platinum Taq, Life Technologies Inc.), but some reduction in product was still evident (data not presented). Other problems when using fluorescent detection were evident and need to be addressed. First, large peaks occasionally obscured nearby, smaller peaks. Separation improvement by changing parameters such as gel concentration, running conditions, etc., should alleviate this problem. However, these approaches have not yet been pursued. Second, a threshold must be established to determine when a peak should be noted as a marker.

Amplifications using newly developed SCAR primers were somewhat unpredictable and needed to be tested both in pairs and multiplexed reactions. Once developed and tested, use of specific primer arrays in multiplex reactions was routine and reliable. The 


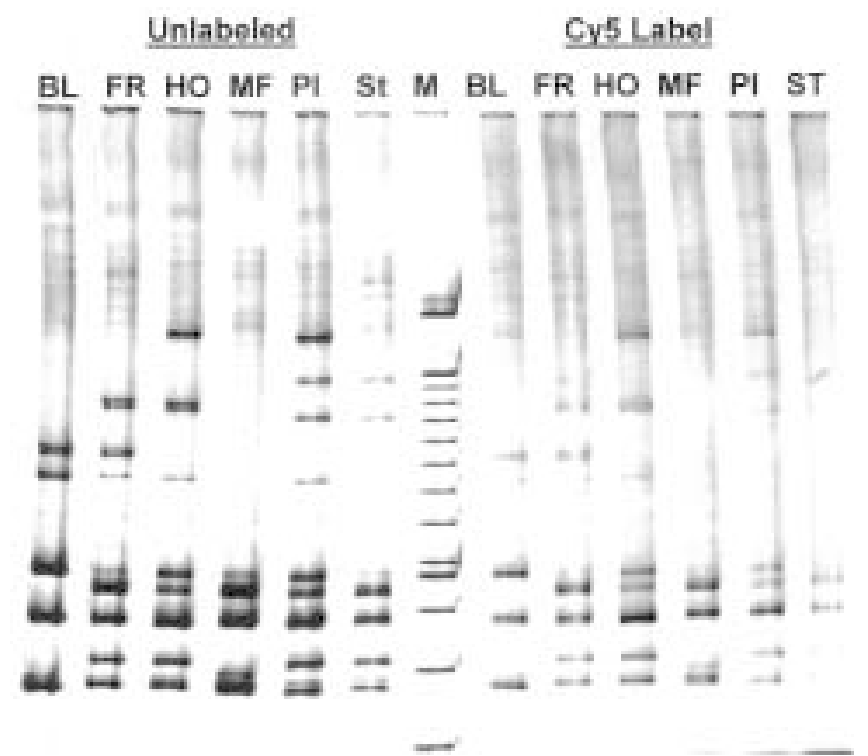

Fig. 4. Silver stained polyacrylamide gel of SCAR fragments amplified using multiplex PCR mix 1 using unlabeled (left side of gel) and Cy5 fluorescent-dye labeled (right side of gel) primers. $M=$ molecular weight marker (100 bp). Cultivar abbreviations are as listed in Table 1.

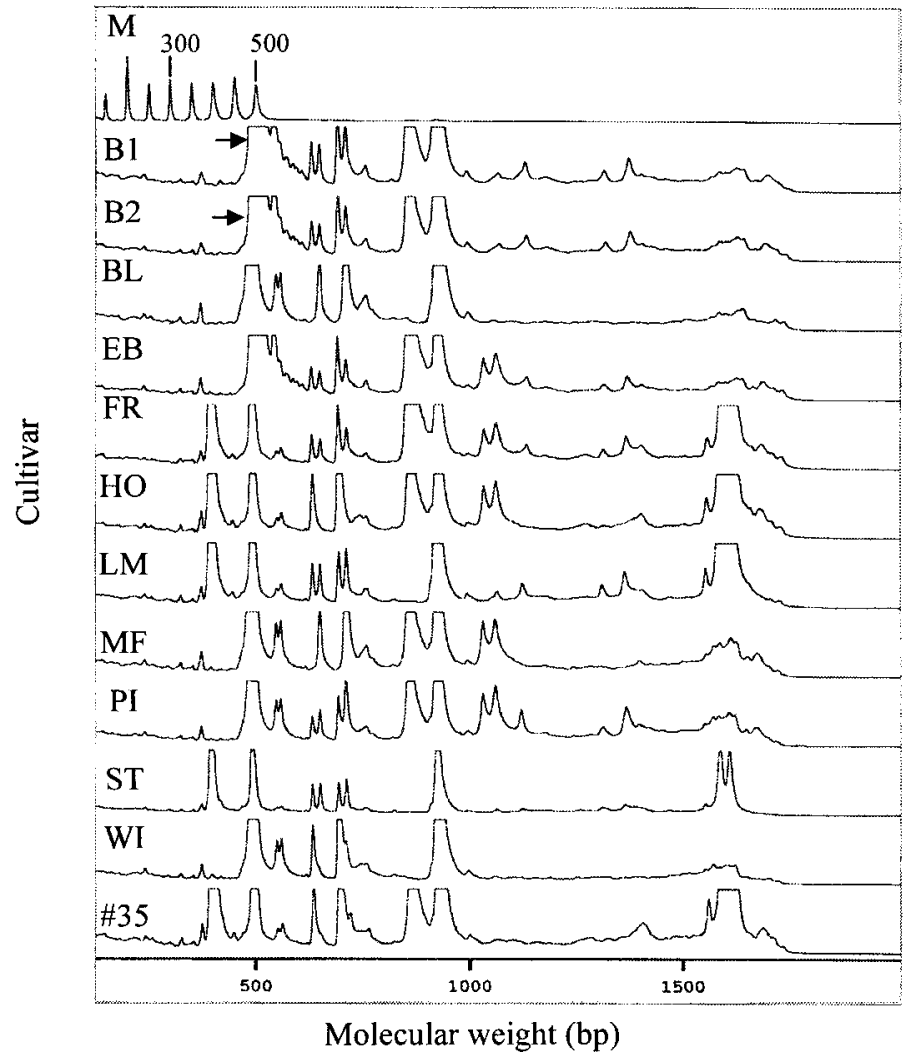

Fig. 5. Chromatogram of Cy5 fluorescent-dye labeled SCAR fragments (using multiplex primer mix 1) separated on a polyacrylamide gel using an AlfExpress automated DNA sequencer (Amersham Pharmacia Biotech, Piscataway, N.J.) and analyzed in Fragment Manager (Amersham Pharmacia Biotech). The size marker (M) peaks are 50 bases apart and range from 150 to $500 \mathrm{bp}$. The 300 - and 500-bp fragments are indicated for reference. Cultivar abbreviations are as detailed in Table 1 with the exception of B1 and B2, which represent two different accessions of the cultivar 'Bergman'. Arrows (in B1 and B2) indicate a large peak that is not properly resolved. advantages of the SCAR marker system outweigh the disadvantage of development time if the markers are required, as in our case, for ongoing analyses. The requirement of only two multiplex reactions reduces reagents and labor used for reaction set up, gel electrophoresis, gel staining, and data interpretation. Since labor is by far the largest expense in this process, any savings in personnel time significantly reduces the per sample cost.

SCAR marker separation efficiency was better than that of RAPD markers based on the number of reactions required per sample. In addition, the SCAR marker gels were cleaner and much easier to score. The clarity and reliability increase with SCAR markers allows greater potential for computer automation. Although some limitations were noted when using the dye $\mathrm{Cy} 5$ for detection, the large number of sample lanes on a typical fluorescent system and the direct computer link to scoring and databasing still makes this detection method a useful alternative to pursue.

\section{Literature Cited}

Amicucci A., C. Guidi, A. Zambonelli, L. Potenza, and V. Stocchi. 2000. Multiplex PCR for the identification of white tuber species. FEMS Microbiol. Lett. 189:265-269.

Ausubel, F.M., R. Brent, R.E. Kingston, D.D. Moore, J.G. Seidman, J.A. Smith, and K. Struhl. 1995. Current protocols in molecular biology. Wiley, New York.

Davin-Regli, A., Y. Abed, R.N. Charrel, C. Bollet, and P.de Micco. 1995. Variations in DNA concentrations significantly affect the reproducibility of RAPD fingerprint patterns. Res. Microbiol. 146:561-568.

Dice, L. R. 1945. Measures of the amount of ecologic association between species. Ecology 26:297-302.

Eck, P. 1990. The american cranberry. Rutgers Univ. Press, New Brunswick, N.J. Hansen, M., C. Hallden, and T. Sall. 1998. Error rates and polymorphism frequencies for three RAPD protocols. Plant Mol. Biol. Rpt. 16:139-146.

Haymes, K.M., W.E.Van de Weg, P. Arens, J.L. Maas, B. Vosman, and A.P.M. Den Nijs. 2000. Development of scar markers linked to a Phytophthora fragariae resistance gene and their assessment in European and North American strawberry genotypes. J. Amer. Soc. Hort. Sci. 125:330-339.

Henegariu, O., N.A. Heerema, S.R. Dlouhy, G.H. Vance, and P.H. Vogt. 1997. Multiplex PCR: Critical parameters and step-by-step protocol. BioTechniques 23:504-511.

Horejsi, T., J.M. Box, and J.E. Staub. 1999. Efficiency of randomly amplified polymorphic DNA to sequence characterized amplified region marker conversion and their comparative polymerase chain reaction sensitivity in cucumber. J. Amer. Soc. Hort. Sci. 124:128-135.

Lynch, M. 1988. Estimation of relatedness by DNA fingerprinting. Mol. Biol. Evolution 5:584-599.

Moscetti, A., I. Boschi, M. Dobosz, G. Destro-Bisol, M. Pescarmona, E. d'Aloja, and V. Pascali. 1995. Fluorescence-based classification of microsatellites using a single-wavelength semiautomatic sequencer: Genotype assignment and identity tests by analysis of comigrating peak profiles. Electrophoresis 16:1875-1880.

Novy, R.G., C. Kobak, J. Goffreda, and N. Vorsa. 1994. RAPDs identify varietal misclassification and regional divergence in cranberry [Vaccinium macrocarpon (Ait.) Pursh]. Theor. Appl. Genet. 88:1004-1010.

Novy, R.G. and N. Vorsa. 1995. Identification of intracultivar genetic heterogeneity in cranberry using silver-stained RAPDs. HortScience 30:600-604.

Novy, R., N. Vorsa, and K. Patten. 1996. Identifying genotypic heterogeneity in 'McFarlin' cranberry: A randomly amplified polymorphic DNA (RAPD) and phenotypic analysis. J. Amer. Soc. Hort. Sci. 121:210-215.

Paran, I. and R.W. Michelmore. 1993. Development of reliable PCR-based markers linked to downy mildew resistance genes in lettuce. Theor. Appl. Genet. 85:985-993. Parent, J.-G. and D. Page. 1998. Identification of raspberry cultivars by sequence characterized amplified region DNA analysis. HortScience 33:140-142.

Perez, T., J. Albornoz, and A. Dominguez. 1998. An evaluation of RAPD fragment reproducibility and nature. Mol. Ecol. 7:1347-1357.

Sarracino, J.M. and N. Vorsa. 1991. Self and cross fertility in cranberry. Euphytica 58:129-136.

Staub, J., J. Bacher, and K. Poetter. 1996. Sources of potential errors in the application of random amplified polymorphic DNAs in cucumber. HortScience 31:262-266.

Stewart, C.N. and L.E. Via. 1993. A rapid CTAB DNA isolation technique useful for RAPD fingerprinting and other PCR applications. Biotechniques 5:748-750. 